Nloman 2021, 39(2)

Revista de Psicologia, Ciències de l'Eduació i de l'Esport

ISSN: $1138-3194$

Facultat de Psicologia, Ciències de l'Educació i de l'Esport Blanquerna

Universitat Ramon Llull

\title{
La mejora de la competencia digital de los futuros docentes de secundaria: una experiencia en la Universidad Miguel Hernández
}

\author{
David Jiménez-Hernández ${ }^{1}$, Víctor González-Calatayud², María Asunción Martínez-Mayoral² \\ \& Javier Morales ${ }^{2}$ \\ ${ }^{1}$ Universidad Nacional de Educación a Distancia, UNED (España) \\ ${ }^{2}$ Universidad Miguel Hernández de Elche (España) \\ Recibido: 2021-6-28 \\ Aceptado: 2021-9-14 \\ https://doi.org/10.51698/aloma.2021.39.2.53-62
}

La mejora de la competencia digital de los futuros docentes de secundaria: una experiencia en la Universidad Miguel Hernández

\begin{abstract}
La competencia digital es una de las competencias clave del sistema educativo. Para su buen desarrollo es necesario que los docentes también tengan adquiridas estas competencias, y, para ello, la formación inicial y permanente son imprescindibles. El principal objetivo fue analizar cómo la formación desarrollada en el máster en Formación del Profesorado de Educación Secundaria Obligatoria y Bachillerato, Formación Profesional y Enseñanza de Idiomas, en la asignatura de TIC para la Docencia y el Aprendizaje, ayuda a mejorar la competencia digital docente de los futuros profesores. Con una escala basada en el modelo DigComp se evaluó la competencia digital antes y después de la formación. La muestra estuvo compuesta por un total de 181 estudiantes (62.98\% eran mujeres). Se analizaron las diferencias encontradas en cada área competencial: se encontró una mejora tras la formación en todas las áreas y se procedió a ajustar un modelo para predecir la probabilidad de que el alumnado alcance una puntuación alta en función de sus características y su nivel de base al inicio. Los resultados evidencian un incremento en todas las áreas, especialmente en la creación de contenidos. El género, la edad y la rama de conocimiento no influyeron en el modelo predictivo, que finalmente se explica en función del nivel competencial inicial del alumnado y del área competencial.
\end{abstract}

Palabras clave: competencia digital; profesorado; educación secundaria; formación del profesorado.

Improving the digital competence of future secondary school teachers: an experience at Miguel Hernández University

\begin{abstract}
Digital competence is one of the key competences in the education system. For students to develop this competence successfully, teachers must also have acquired it. To this end, initial and ongoing training are essential. The aim of the study was to analyse how the training offered in the framework of a Master's Degree in Secondary Education, specifically in the class on ICT for teaching and learning, helps to improve the Digital Teaching Competence of future teachers. Using a scale based on the DigComp model, Digital Competence was assessed before and after the training offered in class. The sample consisted of a total of 181 students, $62.98 \%$ of whom were female. The differences found in each competence area were analysed, with the results showing an improvement after the training in all areas. A model was adjusted to predict the probability of students achieving a high score according to their characteristics and baseline level. The results show an increase in all areas, especially in Content Creation. Gender, age and field of knowledge did not influence the predictive model, which is finally explained as a function of the students' initial competence level and competence area.
\end{abstract}

Keywords: Digital Competence; teachers; Secondary Education; Teacher Education

Correspondencia

Víctor González Calatayud

Universitat d'Elx, Elche, Alicante.

https://orcid.org/0000-0002-6835-0264

victor.gonzalezc@umh.es 


\section{Introducción}

Durante los primeros años del siglo xx, la competencia digital se ha considerado un aspecto básico en la formación de los ciudadanos. De este modo, las universidades se han hecho eco y la han incluido como competencia transversal, prácticamente en todos los niveles educativos, con la llegada de la Convergencia Europea (González-Calatayud et al., 2018) y desde la Declaración de Bolonia de 1999. Uno de los principales objetivos que se ha perseguido con su incorporación ha sido desarrollar un modelo que sea capaz de medir y desarrollar la competencia digital, específicamente de docentes y discentes y, de manera más global, de toda la población (Colás-Bravo et al., 2017). Para llegar a este punto, también es necesario cambiar la manera de afrontar el proceso de enseñanza-aprendizaje, para que dé lugar a enfoques centrados en el alumnado, con métodos didácticos innovadores capaces de formar a ciudadanos críticos y activos (Santos et al. 2017), y que los lleve a introducirse en el mundo digital de manera responsable (Cabero, 2017). Y es que, como pone de manifiesto el informe Educase Horizon (2020), la incorporación de la tecnología en la educación y el uso de metodologías innovadoras es fundamental.

Son varios los modelos que se han elaborado y que Jiménez-Hernández et al. (2021) recogen en su análisis. Uno de los principales modelos sobre competencia digital es el desarrollado por la Comisión Europea, el modelo DigComp (Ferrari, 2013). Este modelo ha servido de base a diversos trabajos que han creado instrumentos alternativos de medición de esta competencia (González-Rodríguez \& Urbina-Ramírez, 2020), así como formación específica para su desarrollo. En su primera versión, este modelo contempló cinco áreas competenciales: información, comunicación, creación de contenidos, seguridad y resolución de problemas. En su última versión (INTEF, 2018), la versión 2.1, se han mantenido las cinco áreas, pero se han modificado los nombres de las áreas competenciales y algunos de los aspectos que se incluían en estas.

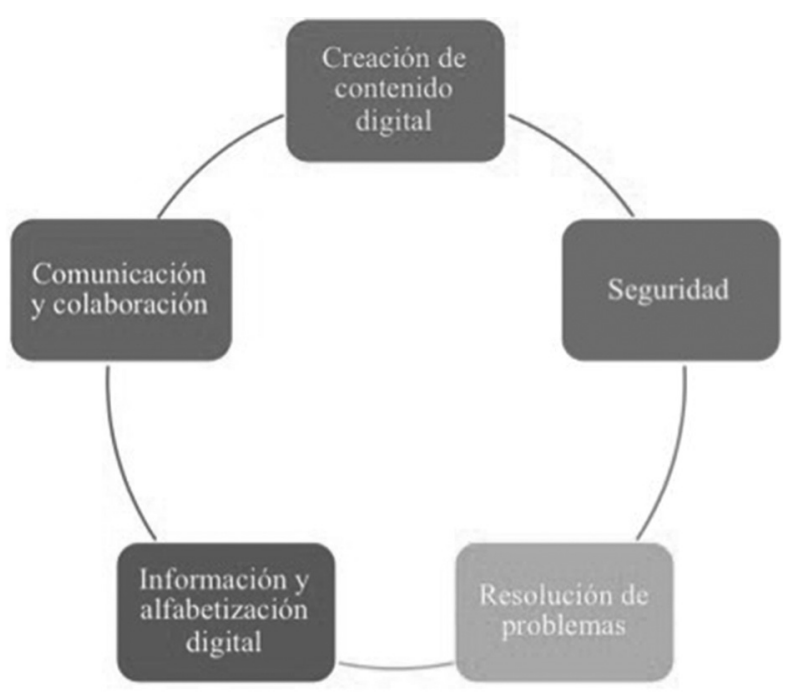

Figura 1. Modelo de la competencia digital del INTEF (2018)
Para definir competencia digital se partirá de la recopilación realizada por Gutiérrez (2011), que la considera: "el conjunto de valores, creencias, conocimientos, capacidades y actitudes para utilizar adecuadamente las tecnologías, incluyendo tanto los ordenadores como los diferentes programas e Internet, que permiten y posibilitan la búsqueda, el acceso, la organización y la utilización de la información con el fin de construir conocimiento" (p. 201). Por el área en la que nos encontramos, se hace ineludible hacer mención también a la definición desarrollada por Krumsvik (2011), por su carácter pedagógico. Krumsvik considera que los docentes deben tener esta competencia adquirida para que les permita usar las TIC en el contexto educativo y en coherencia con unos buenos criterios pedagógicos y didácticos. Además, han de ser conscientes de las implicaciones que supone para el desarrollo de estrategias de aprendizaje y la formación digital del alumnado. Asimismo, por la importancia que entraña esta competencia, el Ministerio de Educación y Formación Profesional (MEFP, 2018) ha considerado que una persona es competente digitalmente cuando tiene conocimientos básicos sobre aplicaciones informáticas (saber), utilización correcta de recursos tecnológicos para resolver problemas en contextos reales (saber hacer), y posesión de actitudes activas, críticas y realistas al emplear los recursos digitales (saber ser).

En este contexto, los docentes se encuentran con una paradoja. Por un lado, las TIC han alcanzado un gran protagonismo en el proceso de enseñanza-aprendizaje y han sido bien recibidas por el profesorado, pero, por otro lado, se experimenta una introducción mínima de las mismas en comparación con la docencia más tradicional. Dicho lo cual, se puede afirmar que esta competencia es una de las más necesarias para el profesorado actual (Cabero, 2014). En nuestro país, la formación en esta temática se ha reclamado desde diferentes instancias, incluyendo la legislativa. De acuerdo con la LOMLOE (Ley Orgánica 3/2020 por la que se modifica la Ley Orgánica de Educación), las competencias clave deben ser trabajadas a través de las diferentes materias. Por eso es necesario que los docentes estén formados y puedan implementarlas en sus aulas.

Actualmente se antoja acuciante que todo docente posea un nivel aceptable de competencia digital, que le permita preparar a su alumnado para los desafíos de la sociedad actual (Esteve, 2015). Sin ir más lejos, nos encontramos en un momento álgido de esta competencia por la situación generada por la pandemia de la COVID-19, que se ha manifestado con el uso apresurado de diferentes recursos y herramientas digitales (Arruti et al., 2020). Para ello, la vía principal es la formación del profesorado mediante TIC, tanto inicial como permanente (Castañeda et al., 2018), que lo capacite para la creación de diferentes contenidos y recursos, de acuerdo con los contextos en los que se encuentre (Salinas et al., 2014), empleando la tecnología correctamente, adaptándola a los aprendizajes que quiera conseguir con su alumnado (Aslan \& Zhu, 2015; Gisbert \& Lázaro, 2015), y favoreciendo el cambio en 
el proceso educativo por medio del pensamiento transdisciplinario y la integración de tecnologías actuales (Mishra et al., 2011). Por lo tanto, tal y como argumentan Durán et al. (2016), cualquier profesional de la educación, sea cual sea su nivel educativo y especialidad, debe alcanzar un nivel básico de competencia digital en el ejercicio de su profesión. Además del cambio en las instituciones educativas para adaptarse digitalmente, dicho cambio debe verse apoyado por una renovación del proceso instructor, encabezada por los docentes para que sea efectiva (López et al., 2019).

Dentro de esta dinámica, es crucial la formación en competencia digital del alumnado del máster en Formación del Profesorado de Educación Secundaria Obligatoria y Bachillerato, Formación Profesional y Enseñanza de Idiomas; alumnado que constituirá el cuerpo docente en un futuro próximo. Dicha formación será el punto inicial de su carrera profesional, por lo que una buena base y concienciación serán clave para continuar desarrollándola a lo largo de la carrera. Así, la formación digital del alumnado universitario está más que justificada según Area (2010) desde dos perspectivas: la académica y la profesional. Desde la académica, que lo capacite para acceder y buscar información, construir conocimiento, comunicarse con nuevos leguajes y con nuevas herramientas y aprender en espacios digitales. Y desde la profesional, para que desarrolle habilidades que lo faculten para un buen cumplimiento profesional. En palabras de Gutiérrez-Porlán et al. (2018), aunque por lo general sea una generación acostumbrada al uso de las tecnologías, el alumnado no aprende mejor con el uso de las TIC por el hecho de utilizarlas en su vida en general. Para llegar a ese punto, hay que trabajar diferentes procesos básicos, como la gestión de información y el desarrollo de habilidades comunicativas. Sobre este último, desde la educación superior se debe formar digitalmente a los futuros docentes para que favorezcan estos aprendizajes en sus futuros estudiantes (Guillén-Gámez \& Perrino, 2020).

\section{Material y método}

\section{Objetivos}

El principal objetivo de esta investigación fue analizar la repercusión del plan formativo impartido en la asignatura TIC para la Docencia y el Aprendizaje del máster en Formación del Profesorado de Educación Secundaria Obligatoria, Bachillerato, Formación Profesional y Enseñanzas de Idiomas de la Universidad Miguel Hernández, en el desarrollo de la competencia digital docente del alumnado. Los objetivos específicos fueron los siguientes:

- Investigar el efecto de la formación en la mejora de la competencia digital en cada una de las áreas competenciales definidas en el modelo DigComp.

- Investigar la relación entre la competencia digital de base al inicio del curso y la evaluada al final tras recibir la formación específica en TIC.
- Investigar el efecto de los condicionantes sociodemográficos del alumnado a la hora de predecir la evolución de su competencia digital.

- Predecir la probabilidad con la que un estudiante conseguirá una competencia digital en un nivel al menos de 4 (lo que implica un nivel notable en una escala del 1 al 5) al finalizar su formación, basándose en las características sociodemográficas relevantes y su nivel inicial de competencia digital.

\section{Desarrollo de la asignatura para trabajar la competencia digital}

El trabajo que aquí se presenta se enmarcó en la formación impartida en la asignatura del máster en Formación del Profesorado de Educación Secundaria Obligatoria y Bachillerato, Formación Profesional y Enseñanza de Idiomas denominada TIC para la Docencia y el Aprendizaje en Secundaria, que el alumnado debía cursar de manera obligatoria, entre los cursos 2017-2018 y 2019 2020. Al iniciar cada curso, el alumnado, en el marco de esta asignatura, realizó un pretest para conocer su nivel de competencia digital docente con el que accedió al máster. Siguiendo el modelo DigCompEdu, elaborado por el INTEF (2017), la asignatura se estructuró para trabajar los contenidos, tanto de manera teórica como práctica, para que el alumnado desarrollara su competencia digital en las cinco áreas competenciales del modelo: (A1) información y alfabetización informacional, (A2) comunicación y colaboración, (A3) creación de contenidos digitales, (A4) seguridad y (A5) resolución de problemas. De este modo, la asignatura se organizó en tres bloques de contenidos, estructurados en ocho temas. Los bloques eran los siguientes: 1) comunicación e información (recursos de comunicación, almacenamiento y colaboración en línea, redes sociales y acceso a la información); 2) recursos y creación de contenidos (blogs, licenciamientos, generación de contenidos y audiovisuales); 3) evaluación (seguimiento y evaluación del aprendizaje).

La asignatura se basó en una metodología eminentemente práctica. El alumnado debía revisar de antemano los contenidos académicos que tenía a su disposición íntegramente antes de ser trabajados en el aula. Al principio de la sesión, el docente realizaba una breve revisión del contenido de cada unidad para, a continuación, desarrollar las actividades propuestas en la evaluación continua. Estas actividades consistían en la realización de seis tareas concretas que el alumnado debía contener en un blog creado por él y parte de la evaluación. La primera tarea consistía en la creación de un perfil profesional en la red social de Twitter, para publicar sus tareas, artículos de interés relacionados con su especialidad y la docencia, y a través del cual debía seguir a profesionales de la educación en su campo. El objetivo último era crear una red de aprendizaje, descubrir propuestas docentes innovadoras y dar a conocer aquello que realizaba para acostumbrarlo a la filosofía de la compartición en abierto (open access). La segunda tarea consistía en la elaboración de un blog que le per- 
Tabla 1. Descripción de actividades y su relación con las áreas competenciales

\begin{tabular}{lll}
\hline Actividades & $\begin{array}{l}\text { Descripción: recursos que hay que } \\
\text { generar o integrar }\end{array}$ & $\begin{array}{l}\text { Áreas } \\
\text { competenciales }\end{array}$ \\
\hline $\begin{array}{l}\text { A1. Redes sociales: } \\
\text { Twitter }\end{array}$ & $\begin{array}{l}\text { Crear y personalizar una cuenta en } \\
\text { Twitter, publicar e interaccionar. }\end{array}$ & A1; A2; A4 \\
\hline A2. Sitio web & $\begin{array}{l}\text { Crear, personalizar y publicar en un } \\
\text { blog de Blogger. Cada alumno } \\
\text { tematizará dicho sitio web como si se } \\
\text { tratara de un blog de la asignatura } \\
\text { vinculada a una materia de su } \\
\text { especialidad en el máster. }\end{array}$ \\
\hline A3. Recursos TIC & $\begin{array}{l}\text { Recopilar recursos TIC de aprendizaje A1; A2; A3; A5 } \\
\text { específicos relacionados con la } \\
\text { materia de la especialidad o con la } \\
\text { actividad de aprendizaje propuesta. }\end{array}$ \\
\hline A4. Presentación & $\begin{array}{l}\text { Crear una presentación de la } \\
\text { actividad de aprendizaje en una } \\
\text { materia o asignatura asociada a la } \\
\text { especialidad del alumno en el máster. }\end{array}$ \\
\hline A5. Vídeo & $\begin{array}{l}\text { Crear y publicar un videotutorial en A2; A3; A5 A2; A3; A5 } \\
\text { formato PechaKucha (20 diapositivas } \\
\text { de 20 segundos cada una), utilizando } \\
\text { la presentación realizada en la } \\
\text { actividad anterior. }\end{array}$ \\
\hline $\begin{array}{l}\text { Crear una rúbrica de evaluación y un A1; A2; A3; A5 } \\
\text { formulario de Google para la } \\
\text { autoevaluación. }\end{array}$ \\
\hline A6. Evaluación
\end{tabular}

mitiera integrar el resto de actividades propuestas. La tercera tarea era la búsqueda, la recopilación y el filtrado de recursos TIC de aprendizaje: diez generales y diez de su especialidad. Tenía que mostrar estos recursos en su blog e indicar en qué consistían y cuál era su uso didáctico. En la cuarta actividad tenía que planificar una actividad docente (basándose en el conocimiento adquirido en la asignatura de Innovación Docente) en la que trabajara los contenidos de su materia con recursos TIC y describiera todos los elementos básicos de una unidad didáctica: contexto, competencias, objetivos, criterios de evaluación, estándares, instrumentos de evaluación, recursos y planificación. La evaluación se realizaba con las presentaciones de Google, que tenía que generar y embeber en el blog, y en la que tenía que incluir imágenes correctamente referenciadas con licencia CC. La quinta actividad que tenía que desarrollar era la elaboración de un vídeo en el que explicara, en formato PechaKucha (20 diapositivas de 20 segundos cada una), la actividad diseñada en la anterior tarea. Este vídeo debía publicarse en abierto y tenía que etiquetarse en YouTube e incrustarlo en el blog. La sexta y última actividad estaba relacionada con la evaluación; el alumnado debía crear una rúbrica de evaluación completa en una hoja de cálculo de Google (con criterios, niveles de evaluación, descripción y pesos) y generar un formulario de Google para registrar la evaluación indicada en la rúbrica.

Como se observa en la tabla 1, el alumnado trabajó todas las áreas competenciales en estas actividades.

Al finalizar la asignatura, el alumnado rellenó el postest de competencia digital docente para evaluar su mejora. La participación en los formularios pretest y postest fue totalmente voluntaria.

\section{Instrumento}

Para evaluar la competencia digital del alumnado en relación con cada una de las áreas competenciales definidas en el modelo DigComp del INTEF se elaboró un cuestionario compuesto por veintiún ítems agrupados en las cinco áreas del modelo. Cada ítem fue evaluado en una escala de puntuación entera entre 1 y 5 , en la que 5 era el máximo nivel de desempeño o capacitación en función de la percepción del estudiante. El número de ítems para evaluar las áreas competenciales A1, A2, A3, A4 y A5 eran, respectivamente, 3, 6, 4, 4 y 4. Para cuantificar la competencia digital en cada una de estas áreas se consideró un índice cuyo valor era el promedio de las valoraciones en los ítems vinculados a dicha área. Además de los ítems propuestos, se recogieron una serie de datos sociodemográficos: género; año de nacimiento, que se categorizó en "nacidos antes / a partir de 1991", dado que ese año es el que divide a la generación pre-Bolonia de la pos-Bolonia; y especialidad cursada en el máster. Esta última variable, dado que eran un total de trece especialidades, se categorizó en los cuatro ámbitos que el currículo de Educación Secundaria definía: científico-tecnológico (ciencias y tecnología), comunicación (lenguas), sociales-humanidades (geografía, historia, economía, plástica) y salud (educación física, orientación educativa y servicios sociocomunitarios).

Para garantizar la fiabilidad del cuestionario utilizado y la confiabilidad de los índices utilizados para describir la competencia digital en cada área competencial, calculamos el alpha de Cronbach con el total de los 812 registros disponibles en las encuestas pretest y postest. Los resultados fueron muy favorables, con valores de alpha de Cronbach superiores a 0.8 en las cinco áreas competenciales y un valor global de 0.95 $(\mathrm{A} 1=.82 ; \mathrm{A} 2=.86 . ; \mathrm{A} 3=.86 ; \mathrm{A} 4=.87 ; \mathrm{A} 5=.86)$. Esto nos permitió asumir la validez de los indicadores competenciales A1, A2, A3, A4 y A5 que construimos con los promedios de las puntuaciones obtenidas en los ítems vinculados a cada área.

\section{Participantes}

El número de respuestas disponibles entre los cursos 2017-2018 y 2019-2020 fue de 812, 505 de los cuales correspondían a respuestas al inicio del curso y 307 al final. Tan solo 181 sujetos respondieron tanto al inicio como al final, por lo tanto, solo con ellos se llevó a cabo el análisis de la evolución de la competencia digital docente. La distribución de la muestra disponible de 181 estudiantes respecto a las variables sociodemográficas consideradas se presenta en la tabla 2.

Tabla 2. Datos sociodemográficos

\begin{tabular}{lllll}
\hline & $\begin{array}{l}\text { Científico- } \\
\text { tecnológico }\end{array}$ & $\begin{array}{l}\text { Social- } \\
\text { humanidades }\end{array}$ & Salud & Comunicación \\
\hline Ámbito & $39.23 \%$ & $29.83 \%$ & $13.81 \%$ & $17.13 \%$ \\
\cline { 2 - 5 } & $1964-1990$ & & $1991-1997$ & \\
\hline Época & $56.35 \%$ & & $43.65 \%$ & \\
\cline { 2 - 4 } & Mujer & & Hombre & \\
\hline Género & $62.98 \%$ & & $37.02 \%$ & \\
\hline
\end{tabular}




\section{Análisis de datos}

El preprocesado de los datos tuvo en cuenta las incoherencias detectadas cuando las puntuaciones finales en competencia digital (tras la formación) eran inferiores a las iniciales. Estas incoherencias repercutirían en sesgos notables que se trató de reducir parcialmente nivelando a cero la mejora de estos sujetos, y por lo tanto, asumiendo que la formación no tuvo efecto alguno en la competencia digital que manifestaron al finalizar el curso. Puesto que se trabajó con percepciones personales, resultó más creíble la valoración final del sujeto tras haber adquirido mayor conocimiento específico de la materia respecto de la que valoraba sus habilidades y capacidades. En estos casos, las valoraciones iniciales fueron sustituidas, pues, por las finales.

Los descriptivos utilizados consistían en frecuencias (porcentajes) para las variables cualitativas y gráficos de cajas (box plot, representando cuartiles) para las valoraciones de los indicadores con las puntuaciones en cada área competencial.

Para testar si el nivel competencial digital docente aumentaba al finalizar el curso, se utilizó el test no paramétrico de Wilcoxon para muestras pareadas, para comparar las puntuaciones iniciales con las finales bajo la hipótesis alternativa de que había aumentado.

Puesto que el objetivo planteado era predecir la probabilidad de alcanzar un grado de competencia digital "notable", se generó una nueva variable con valores $1 / 0$ para identificar cuándo un sujeto proporcionaba una valoración mayor o igual a 4 en cada uno de los índices. Esta variable se introdujo como variable respuesta en un modelo logit binomial, y se consideraron las variables sociodemográficas descritas anteriormente como posibles variables explicativas, junto con el área competencial y la valoración inicial obtenida en cada área.

Puesto que cada sujeto proporcionó un total de 10 valoraciones promedio ( 5 al inicio del curso -una por cada área competencial- y 5 al final), y todas estaban relacionadas entre sí (no eran independientes), a la hora de plantear un modelo de predicción, era preciso introducir un efecto aleatorio que identificara a cada sujeto y vinculara todas sus respuestas. El análisis estadístico se realizó utilizando el software R y la interfaz R-Studio.

El procedimiento de selección de variables en el modelo propuesto, y por lo tanto el descarte de las que no resultaban relevantes para predecir la respuesta, se realizó según el criterio AIC (Akaike, 1974), que tenía en cuenta la bondad del ajuste y la complejidad del modelo (penalizaba modelos complejos). Se priorizaron los modelos con un menor AIC.

\section{Resultados}

\section{Análisis descriptivo}

En la figura 2 se muestran las puntuaciones en los índices de competencia digital al inicio (gráfico izquierdo) y al final del curso (gráfico derecho). En el eje horizon-
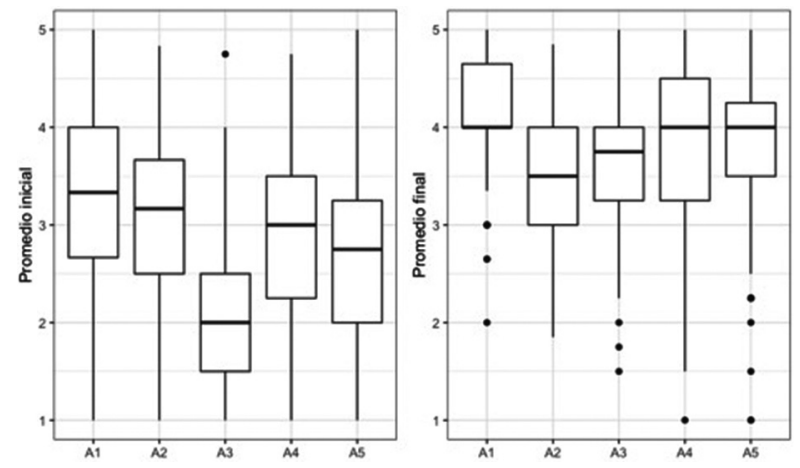

Figura 2. Diagrama de caja de promedio de puntuaciones en las áreas competenciales al inicio (izquierda) y al final (derecha).

tal se representan las cinco áreas de competencia digital. Las cajas están delimitadas por los cuartiles primero y tercero y la línea central representa la mediana, esto es, el valor que separa al 50\% de observaciones a un lado y el resto, al otro. Los bigotes se extienden entre los valores mínimo y máximo, de modo que obtenemos información sobre la localización de la mayoría de los datos (en torno a la mediana) y su dispersión (la amplitud de los bigotes).

Observamos que, inicialmente, los sujetos tenían más desarrolladas las competencias digitales vinculadas a las áreas A1 (información) y A2 (comunicación), y especialmente poco desarrolladas las vinculadas al área A3 (creación de contenidos digitales). Al final del curso todas las puntuaciones crecieron y llegaron generosamente a niveles de 4 para A1, y en torno a 4 (coincide con la mediana) para A4 (seguridad) y A5 (resolución de problemas). Las puntuaciones relacionadas con A2 no crecieron comparativamente tanto como en el resto de áreas, y el crecimiento en A3 también fue sustancial (la mediana varía de 2 a 3.8 desde el inicio hasta el final del curso).

$\mathrm{Al}$ realizar el test de Wilcoxon para muestras pareadas en cada una de las áreas competenciales, en todas se ratificó el efecto de la formación sobre el aumento del nivel competencial respecto del nivel manifestado al inicio del curso. Todos los p-valores fueron significativos al $1 \%$ (inferiores a $2.2 \cdot 10^{-16}$ ).

\section{Modelización}

El modelo de predicción propuesto relaciona la probabilidad $(p)$ de obtener una valoración competencial (puntuación final, $p f$ ) al final del curso de al menos 4 , $p=\operatorname{Pr}(p f=4)$, en función de la puntuación inicial ( $p i)$, el área competencial $(A)$, la interacción entre ambas (I:A), y las variables sociodemográficas recopiladas: edad $(E)$, género $(G)$, ámbito de especialidad $(A m b)$. Vinculamos todas las observaciones del mismo sujeto a través de un efecto aleatorio (ID). Relacionar de modo lineal estas variables con la respuesta es habitual, dado que se trata de una probabilidad entre 0 y 1 , con una transformación logit. El modelo propuesto inicialmente es, pues, el siguiente: $\operatorname{logit}(p) \sim p i+A+p i: A+E+G$ $+A m b+I D$, donde $\operatorname{logit}(p)=\exp (p) /(1+\exp (p))$ y $\exp ()$ representa la función exponencial. 
Tabla 3. Modelos ajustados y valor del AIC

\begin{tabular}{ll}
\hline Modelo & AIC \\
\hline M1: logit $(p) \sim p i+A+p i: A+I D$ & 792.23 \\
\hline M2: logit $(p) \sim p i+A+p i: A+G+I D$ & 792.86 \\
\hline M3: logit $(p) \sim p i+A+p i: A+E+I D$ & 793.98 \\
\hline M4: logit $(p) \sim p i+A+p i: A+A m b+I D$ & 793.02 \\
\hline M5: logit $(p) \sim p i+A+p i: A+E+G+I D$ & 794.80 \\
\hline M6: logit $(p) \sim p i+A+p i: A+E+G+A m b+I D$ & 796.07 \\
\hline
\end{tabular}

Tabla 4. Modelo de predicción de un nivel competencial al menos de 4 en cada área

\begin{tabular}{ll}
\hline Área competencial & Modelo de predicción \\
\hline $\mathrm{A} 1$ & $p=\exp (-3.85+2.23 \mathrm{pi}) /(1+\exp (-3.85+2.23 \mathrm{pi}))$ \\
\hline $\mathrm{A} 2$ & $p=\exp (-26.94+7.47 \mathrm{pi}) /(1+\exp (-26.94+7.47 \mathrm{pi}))$ \\
\hline $\mathrm{A} 3$ & $p=\exp (-3.83+1.48 \mathrm{pi}) /(1+\exp (-3.83+1.48 \mathrm{pi}))$ \\
\hline $\mathrm{A} 4$ & $p=\exp (-5.46+2.03 \mathrm{pi}) /(1+\exp (-5.46+2.03 \mathrm{pi}))$ \\
\hline $\mathrm{A} 5$ & $p=\exp (-4.04+1.66 \mathrm{pi}) /(1+\exp (-4.04+1.66 \mathrm{pi}))$ \\
\hline
\end{tabular}

En la tabla 3 se muestran los distintos modelos plausibles ajustados, incluyendo y excluyendo variables sociodemográficas (excepto ID), junto con el AIC resultante. El mejor modelo de todos los propuestos (con menor AIC, 792.23) es el siguiente: $\operatorname{logit}(p) \sim p i+A+i p: A$ $+I D$, de manera que se concluye que la probabilidad de éxito al final de curso, esto es, de conseguir una puntuación competencial al menos de 4 puntos (en una escala del 1 al 5), es diferente para cada área competencial $(A)$ y también influye de modo distinto en cada área competencial, la puntuación inicial (pi). El resto de variables quedaron excluidas como poco relevantes.

Este modelo, además de corroborar el efecto de la formación en el desarrollo de la competencia digital de los sujetos (la puntuación inicial siempre afecta de modo positivo en la respuesta, incrementando la probabilidad de conseguir un nivel competencial notable, como se aprecia en los coeficientes de la tabla 5), nos permite predecir la probabilidad de que un sujeto con una puntuación inicial pi en una determinada área competencial $A$ alcance un nivel competencial al menos de 4 al finalizar el curso. Los modelos de predicción en cada una de las áreas competenciales se muestran en la tabla 4.

En la figura 3 se muestra las curvas de predicción resultantes para cada una de las áreas competenciales. Se aprecia que, puesto que la mayoría de los sujetos partía de una competencia digital media en el área A2 (pi en torno a 3), la probabilidad de incrementar la competencia a nivel $4(p>0)$ solo se inicia para valores de $p i$ superiores a 3, como es de esperar. En la tabla 5 se proporcionan las predicciones (junto con sus intervalos de confianza al 95\%) de la probabilidad de alcan-

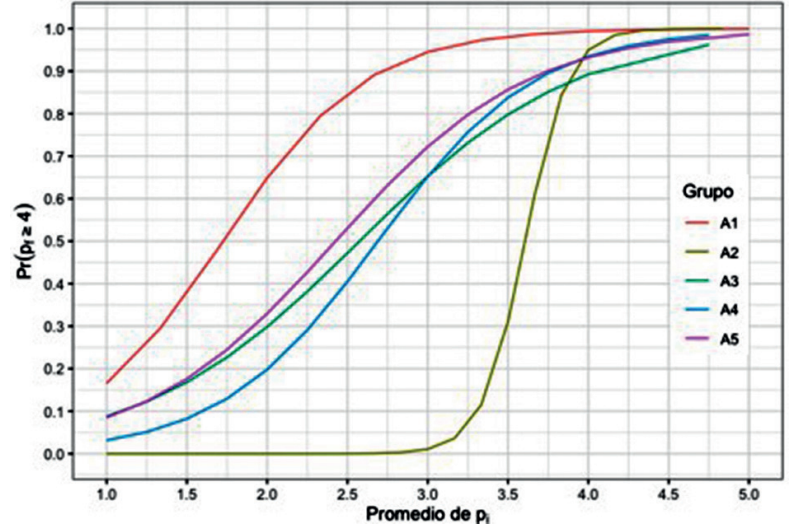

Figura 3. Curvas de predicción de la probabilidad de conseguir una puntuación competencial al menos de 4 tras la formación.

zar el nivel 4 al finalizar el curso, en función de distintos valores para la puntuación inicial.

A modo de verificación de la efectividad del modelo, se calcula la tasa de clasificación correcta según el modelo, que resulta ser del 90.5\%; esto es, el 90.5\% de los datos en cualquier área competencial es correctamente clasificado con una probabilidad superior al 50\% de alcanzar un nivel competencial final de 4, cuando realmente lo alcanzó tras finalizar su formación en el máster.

\section{Discusión y conclusiones}

El uso de las TIC es tan generalizado en la sociedad que, actualmente, como indican Centeno y Cubo (2013), es difícil encontrar estudiantes que no posean alguna competencia en el manejo de las mismas. Sin embargo, esa competencia no siempre se relaciona con el aprendizaje y, mucho menos, con la posibilidad de utilizarlas para la enseñanza. Es por ello por lo que la formación en el uso de las TIC, tanto inicial como formativa, se vuelve imprescindible actualmente (Castañeda et al., 2018). Esta formación ha resultado de gran utilidad según demuestran ciertas investigaciones (GonzálezCalatayud et al., 2018; Gutiérrez \& Serrano, 2016; Romero et al., 2017). Partiendo de este punto, en la asignatura TIC para la Docencia y el Aprendizaje en Secundaria del máster en Formación del Profesorado de Educación Secundaria Obligatoria y Bachillerato, Formación Profesional y Enseñanza de Idiomas se propuso conocer si la formación aportada repercutía en la percepción de la competencia digital de los futuros docentes.

En primer lugar, se ha podido comprobar que la percepción de la competencia digital inicial que tenía el alumnado, exceptuando el área de creación de con-

Tabla 5. Probabilidad de alcanzar una puntuación final de 4 al terminar el curso en función de la puntuación competencial inicial pi, en cada una de las áreas competenciales

\begin{tabular}{llllll}
\hline pi & A1 & A2 & A3 & A4 & A5 \\
\hline 1 & $0.17[0.03,0.55]$ & $0.00[0.00,0.00]$ & $0.09[0.04,0.20]$ & $0.03[0.01,0.11]$ & $0.08[0.03,0.23]$ \\
\hline 2 & $0.65[0.40,0.84]$ & $0.00[0.00,0.00]$ & $0.30[0.20,0.42]$ & $0.20[0.10,0.34]$ \\
\hline 3 & $0.95[0.89,0.97]$ & $0.01[0.00,0.04]$ & $0.65[0.47,0.80]$ & $0.65[0.52,0.76]$ & $0.33[0.20,0.48]$ \\
\hline 4 & $0.99[0.98,1.00]$ & $0.95[0.82,0.99]$ & $0.89[0.68,0.97]$ & $0.93[0.84,0.97]$ & $0.93[0.83,0.98]$ \\
\hline
\end{tabular}


tenidos digitales, donde resultó más bajo, fue un nivel medio, cercano al 3 sobre 5 . Resultados similares a los obtenidos por Romero et al. (2017), cuya utilización general de las TIC fue media o, Gutiérrez y Serrano (2016), que tenían una percepción sobre su competencia más alta, principalmente en información, comunicación y resolución de problemas.

Después de la formación impartida, teniendo en cuenta el material ofrecido, así como las actividades propuestas, se comprobó que el promedio en cada una de las áreas estuvo en torno o por encima de 4 . El incremento en el promedio obtenido no fue muy grande en el área de comunicación, aunque sí significativo, ya que en esta el nivel inicial ya tenía una media más alta. Este hecho contrasta con lo encontrado por GonzálezCalatayud et al. (2018), los cuales hallaron un menor incremento en el área de resolución de problemas. Que el área de comunicación es en la que están más formados los futuros docentes, ya fue comprobado por otras investigaciones (Cózar \& Roblizo, 2014; Prendes et al., 2010), que mostraron su dominio en diferentes herramientas web para la búsqueda de información y comunicación. Además, los estudiantes en Gutiérrez-Porlán et al. (2018) también utilizaban herramientas básicas, como el correo electrónico o las redes sociales con fines comunicativos. El área en el que más aumentó el nivel competencial fue en la creación de contenidos -que pasó de un 2 a un 3.8-, lo que concuerda con otras investigaciones actuales (González-Calatayud et al., 2018; Gutiérrez \& Serrano, 2016). De forma general se puede concluir que la formación aportada en relación con las TIC para la docencia ayuda a mejorar el nivel competencial digital de los futuros docentes tal y como ellos lo perciben.

Gutiérrez y Serrano (2016) también obtuvieron unos resultados similares, aunque sus estudiantes percibieron menos mejora en el área de seguridad después de la formación recibida en su asignatura. Todo lo expuesto nos permite afirmar lo mismo que Gisbert y Esteve (2011), es decir, en el nivel universitario, los estudiantes ya disponen de un nivel de competencia digital básico, pero necesitan formación para llegar a un nivel aceptable para emplearlo en el ámbito educativo. Seguramente sea esta la razón por la que el área de creación de contenidos sea la que más crezca después de la formación. Estamos de acuerdo con Gutiérrez et al. (2010) en remarcar la importancia de la formación del futuro profesorado en competencias digitales para que después repercuta en su aplicación en el resto de niveles educativos.

En la segunda parte de la investigación ha quedado patente que, finalmente, ninguna de las variables sociodemográficas estudiadas (género, edad y rama de conocimiento) ha tenido influencia en el modelo planteado para explicar la consecución de un nivel notable en la competencia digital docente.

Sobre el género, hay estudios sobre una mayor utilización de recursos TIC para cualquiera de los dos. Cózar y Roblizo (2014) y Torres-Coronas y Vidal-Blasco (2015) comprobaron que las mujeres usaban más estos medios; en Gutiérrez et al. (2018) empleaban más herramientas, como las redes sociales; en cambio, según Gil-Fernández et al. (2019), no había diferencia en cuanto al empleo de las redes; mientras que GuillénGámez y Perrino (2020) afirman que es mayor esta competencia en los hombres. Por lo que respecta al profesorado, también se continuaba con esta tendencia (Cabanillas et al., 2020; Riquelme, 2019; Solís \& Jara, 2019).

Al igual que los resultados encontrados en otras investigaciones (Falcó, 2017; Fernández-Cruz \& Fernández-Díaz, 2016; García et al., 2012; García et al., 2016; Romero et al., 2017; Suárez-Rodríguez et al., 2021) no se obtuvieron diferencias significativas en función del género, aunque en el caso de García et al. (2012) sí observaron que los hombres utilizaban más este tipo de herramientas para actividades lúdicas y las mujeres lo hacían más para aspectos sociales y comunicativos.

En lo referente a la edad existen diferentes estudios que van en sintonía con lo expuesto y que confirman que no influyen en la competencia digital del alumnado (Falcó 2017; Gisbert \& Esteve, 2011; Moreno-Rodríguez et al., 2018; Pozuelo, 2014; Prendes \& Román, 2017; Romero \& Minelli, 2011), por lo tanto, no se puede afirmar que los nativos digitales sean más competentes que el resto. Todo ello contrasta con Cózar y Roblizo (2014), en cuya investigación los estudiantes más jóvenes tenían un mejor dominio, al considerar que desde edades más tempranas tuvieron acceso a recursos tecnológicos.

Como en las anteriores variables, la rama de conocimiento no influyó en la competencia digital, y se observa una homogeneidad parecida a la de Gutiérrez et al. (2018). A la inversa que García et al. (2012), que sí comprobaron que los estudiantes del área de ciencias naturales y técnicas tenían una competencia digital mayor que los de ciencias sociales y humanidades. Torres-Coronas y Vidal-Blasco (2015) también encontraron diferencias significativas en la competencia digital según esta variable, aunque no para todas sus dimensiones. En una muestra de docentes, FernándezCruz y Fernández-Díaz (2016) comprobaron que los de primaria y secundaria del área científico-tecnológica mostraban mejor competencia digital que los procedentes de otras ramas. En el caso de Falcó (2017), aunque no encontró diferencias relacionadas con las áreas en las que impartían docencia, sí encontró la excepción en el mayor uso de estas herramientas en el área de artes y oficios, música y tecnología.

El objetivo final del estudio fue la elaboración de un modelo para predecir la probabilidad de que un estudiante obtuviera una puntuación al menos 4 de media, que se puede considerar como un nivel notable, en su percepción sobre su propia competencia digital. Después de la realización de distintas alternativas de modelización con las variables sociodemográficas disponibles, se ha podido comprobar que el mejor modelo de predicción es aquel que considera únicamente la puntuación inicial del alumnado, el área competencial y la interacción entre estas dos variables. Por lo tanto, 
como ya se ha mencionado, se descarta que el género, la edad y la rama de conocimiento afecten al alumnado a la hora de conseguir un nivel competencial notable tras su formación. El modelo ajustado permite, a su vez, predecir la probabilidad de alcanzar niveles competenciales digitales docentes destacables, partiendo exclusivamente del nivel competencial de base del estudiante al inicio del curso. El incremento competencial significativo en cada una de las áreas competenciales permite, además, afirmar que la formación es esencial para mejorar y así alcanzar un nivel aceptable en la competencia digital docente (Durán et al., 2016).

Por lo tanto, se puede concluir que la formación aportada durante el desarrollo del máster en la asignatura de TIC para la Docencia y el Aprendizaje contribuye positivamente a mejorar la competencia digital de los futuros docentes, según la propia percepción del alumnado, y que dicha mejora es predecible exclusivamente en función de su nivel inicial al comenzar el curso. Por ende, como Castañeda et al. (2018) y Salinas et al. (2014) afirmaron, la formación es fundamental para que los docentes mejoren su competencia digital.

Declaración de divulgación de los autores: No existen intereses en conflicto

\section{Referencias}

Akaike, H. (1974). A new look at the statistical model identification. IEEE, Transactions on Automatic Control, 19(6), 716-723. http://doi.org/10.1109/ TAC.1974.1100705

Area, M. (2010). Why Offer Information and Digital Competency Training in Higher Education? RUSC, Revista de Universidad y Sociedad del Conocimiento, 7(2), 2-5. http://dx.doi.org/10.7238/rusc.v7i2.976

Arruti, A., Paños-Castro, J., \& Korres, O. (2020). Análisis de contenido de la competencia digital en distintos marcos legislativos. Aloma: revista de psicologia, ciències de l'educació i de l'esport Blanquerna, 38(2), 149-156. https://doi.org/10.51698/aloma.2020.38.2.149-156

Aslan, A., \& Zhu, C. (2015). Pre-service teachers' perceptions of ICT integration in teacher education in Turkey. Turkish Online Journal of Educational Technology, 14(3), 97-110. http://www.tojet.net/articles/ v14i3/14310.pdf

Cabanillas, J.L., Luengo, R., \& Torres, J.L. (2020) La búsqueda de información, la selección y creación de contenidos y la comunicación docente. RIED. Revista Iberoamericana de Educación a Distancia, 23, 241267. https://doi.org/10.5944/ried.23.1.24128

Cabero, J. (2014). University teacher training in ICT. Application of Delphi method for the selection of training content. Educación XX1, 17(1), 111-132. https://doi.org/10.5944/educxx1.17.1.10707

Cabero, J. (2017). La formación en la era digital: ambientes enriquecidos por la tecnología. Revista Gestión de la Innovación en Educación Superior, 2(2), 41-64. http://hdl.handle.net/11441/67192
Castañeda, L., Esteve, F., \& Adell, J. (2018). Why rethinking teaching competence for the digital world? Revista de Educación a Distancia, 56, 1-20. http:// dx.doi.org/10.6018/red/56/6

Centeno, G. y Cubo, S. (2013). Evaluación de la competencia digital y las actitudes hacia las TIC del alumnado universitario. Revista de Investigación Educativa, 31(2), 517-536. http://dx.doi.org/10.6018/ rie.31.2.169271

Colás-Bravo, P., Conde-Jiménez, J., \& Reyes-de-Cózar, S. (2017). Competencias digitales del alumnado no universitario. RELATEC. Revista Latinoamericana de Tecnología Educativa, 16(1), 7-20. http://dx.medra. org/10.17398/1695-288X.16.1.7

Cózar, R., \& Roblizo, M. J. (2014). La competencia digital en la formación de los futuros maestros: percepciones de los alumnos de los Grados de Maestro de la Facultad de Educación de Albacete. RELATEC: Revista Latinoamericana de Tecnología Educativa, 13(2), 119-133.

Durán, M., Gutiérrez, I., \& Prendes, M. P. (2016). Análisis conceptual de modelos de competencia digital del profesorado universitario. RELATEC: Revista Latinoamericana de Tecnología Educativa, 15(1), 97-114. http://doi.org/10.17398/1695288X.15.1.97

Esteve, F. (2015). La competencia digital docente: análisis y evaluación del desempeño de los estudiantes universitarios de educación por medio de un entorno 3D (Tesis Doctoral). Universitat Rovira i Virgili. http://francescesteve.es/tesis/

Falcó, J. M. (2017). Assessment of Digital Competence in Teachers in the Autonomous Community of Aragon. Revista Electrónica de Investigación Educativa, 19, $73-83$. https://doi.org/10.24320/redie.2017.19.4.1359

Fernández-Cruz, F., \& Fernández-Díaz, M.J. (2016). Generation Z's teachers and their digital skills. Comunicar, 46, 97-105. https://doi.org/10.3916/C462016-10

Ferrari, A. (2013). DIGCOMP: A framework for developing and understanding digital competence in Europe. http:// ftp.jrc.es/EURdoc/JRC83167.pdf

García, I., Gros, B., \& Escofet, A. (2012). La influencia del género en la cultura digital del estudiantado universitario. Athenea Digital, 12(3), 95-114. https:// doi.org/10.5565/rev/athenead/v12n3.1075

García, R., Rebollo, Á., \& García, C. (2016). Relación entre las preferencias de formación del profesorado y su competencia digital en las redes sociales. Bordón. Revista de Pedagogía, 68, 137-153. https://doi. org/10.13042/Bordon.2016.68209

Gil-Fernández, R., Calderón-Garrido, D., León-Gómez, A., \& Martin-Piñol, C. (2019). Comparativa del uso educativo de las redes sociales en los grados de Maestro: universidades presenciales y online. Aloma: revista de psicologia, ciències de l'educació i de l'esport Blanquerna, 37(2), 75-81. https://doi.org/10.51698/ aloma.2019.37.2.75-81

Gisbert, M., \& Esteve, F. (2016). Digital Leaners: la competencia digital de los estudiantes universitarios. 
La cuestión universitaria, 7(1), 48-59. http://polired. upm.es/index.php/lacuestionuniversitaria/article/ view/3359/3423

Gisbert, M., \& Lázaro, J. L. (2015). Professional development in teacher digital competence and improving school quality from the teachers' perspective: a case study. NAER, New Approaches in Educational Research, 4(2), 115-122. https://doi.org/10.7821/ naer.2015.7.123

González-Calatayud, V., Román, M., \& Prendes, M. P. (2018). Formación en competencias digitales para estudiantes universitarios basada en el modelo DigComp. Edutec. Revista electrónica de tecnología educativa, 65, 1-15. https://doi.org/10.21556/edutec.2018.65.1119

González-Rodríguez, C., \& Urbina-Ramírez, S. (2020). Análisis de instrumentos para el diagnóstico de la competencia digital. Revista interuniversitaria de investigación en Tecnología Educativa, 9, 1-12. http:// dx.doi.org/10.6018/riite.411101

Guillén-Gámez, F. D., \& Perrino, M. (2020). Análisis Univariante de la Competencia Digital en Educación Física: un estudio empírico. Retos, 37, 326-332. https://doi.org/10.47197/retos.v37i37.72052

Gutiérrez, A., Palacios, A., \& Torrego, L. (2010). School Teacher Training and ICT Integration in Education: Anatomy of a Mismatch. Revista de Educación, 352, 1-17. http://www.revistaeducacion.educacion.es/ re352/re352_TIC.pdf

Gutiérrez, I. (2011). Competencias del profesorado universitario en relación al uso de tecnologías de la información y comunicación: Análisis de la situación en España y propuesta de un modelo de formación (Tesis Doctoral). Universidad Rovira i Virgili. http://hdl.handle. net/10803/52835

Gutiérrez, I. \& Serrano. J. L. (2016). Evaluation and development of digital competence in future primary school teachers at the University of Murcia. Journal of New Approaches in Educational Research, 5(1), 51-56. http://doi.org/10.7821/naer.2016.1.152

Gutiérrez-Porlán, I., Román-García, M., \& SánchezVera, M. M (2018). Strategies for the communication and collaborative online work by university students. Comunicar, 26(54), 91-100. https://doi.org/10.3916/ C54-2018-09

INTEF (2018). Marco común de Competencia Digital Docente. https://aprende.intef.es/sites/default/files/2018-05/2017_1020_Marco-Com\%C3\%BAn-deCompetencia-Digital-Docente.pdf

Jiménez-Hernández, D., Muñoz, P., \& Sánchez, F. (2021). La Competencia Digital Docente, una revisión sistemática de los modelos más utilizados. Revista interuniversitaria de investigación en Tecnología Educativa, 10, 105-120. https://doi.org/10.6018/riite. 472351

Krumsvik, R. J. (2011). Digital competence in the Norwegian teacher education and schools. Högre utbildning, 1(1), 39-51. https://hogreutbildning.se/ index.php/hu/article/download/874/1817

Ley Orgánica 3/2020, de 29 de diciembre, por la que se modifica la Ley Orgánica 2/2006, de 3 de mayo, de Educación (LOMLOE). Boletín Oficial del Estado (BOE), 340, de 30 de diciembre de 2020, 122868 a 122953. https://www.boe.es/boe/dias/2020/12/30/ pdfs/BOE-A-2020-17264.pdf

López, J., Pozo, S., Fuentes, A., \& Romero, JM. (2019). Análisis del Liderazgo Electrónico y la Competencia Digital del Profesorado de Cooperativas Educativas de Andalucía (España). Multidisciplinary Journal of Educational Research, 9(2), 194-223. http://doi. org/10.4471/remie.2019.4149

MEFP (2018). Ministerio de Educación y Formación Profesional del Gobierno de España. http://www.educacionyfp.gob.es/educacion/mc/lomce/elcurriculo/

Mishra, P., Koehler, M., \& Henriksen, D. (2011). The seven trans-disciplinary habits of mind: Extending the TPACK framework towards 21st century learning. Educational Technology, 11(2), 22-28. https://citeseerx. ist.psu.edu/viewdoc/download?doi=10.1.1.701.429 $3 \&$ rep=rep1\&type $=$ pdf

Moreno-Rodríguez, M.D., Gabarda-Méndez, V., \& Rodríguez-Martín, A.M. (2018). Informational literacy and digital competence in teacher education students. Profesorado. Revista Currículum y Formación del Profesorado, 22, 253-270. https://doi.org/10.30827/ profesorado.v22i3.8001

Prendes, M. P., Castañeda, L., \& Gutiérrez, I. (2010). ICT competences of future teachers. Comunicar, 18(35), 175-182. http://doi.org/10.3916/C35-201003-11

Prendes, M.P., \& Román, M.M. (2017). Entornos Personales de Aprendizaje: Una Visión Actual de Cómo Aprender con Tecnologías. Octaedro

Riquelme, I. (2019). Comparación de las competencias digitales docentes en una universidad tecnológica en Chile desde una perspectiva de género. En E. SánchezRivas, J. Ruiz-Palmero y E. Sánchez (Eds.), Innovación y tecnología en Contextos Educativos (pp. 25-35). UMA Editorial

Romero, M., \& Minelli, J. (2011). The Wobbly Net Generation: student teachers' perception of their ICT Skills. Education in the Knowledge society, 12(3), 280298. https://doi.org/10.14201/eks.8492

Romero-Martín, R., Castejón-Oliva, F. J., López-Pastor, V. M., \& Fraile-Aranda, A. (2017). Formative assessment, communication skills and ICT in Initial teacher education. Comunicar, 25(52), 73-82. https:// doi.org/10.3916/C52-2017-07

Pozuelo, J. Y. (2014). ¿Y si enseñamos de otra manera? Competencias digitales para el cambio metodológico. Caracciolos. Revista Digital de Investigación en Docencia, 2(1), 1-21.

Salinas, J., De Benito, B., \& Lizana, A. (2014). Competencias docentes para los nuevos escenarios de aprendizaje. Revista Interuniversitaria de Formación del Profesorado, 78(28.1), 145-163. http://hdl.handle. net/11162/111548

Santos, M. A., Sotelino, A., Jover, G., Naval, C., Álvarez, J. L., \& Vázquez, V. (2017). Design and validation of a questionnaire on university teaching practice and 
attitude towards innovation (CUPAIN). Educación XX1, 20(2), 39-71. https://doi.org/10.5944/eduCXx1.19031

Solís, J, \& Jara, V. (2019). Competencia digital de docentes de Ciencias de la Salud de una universidad chilena. Píxel-Bit. Revista Medios Educación, 56, 193211. https://doi.org/10.12795/pixelbit.2019.i56.10 Suárez-Rodríguez, J.M., Almerich, G., Díaz-García, I., \& Fernández-Piqueras, R. (2012). ICT Competences of teachers. Influence of personal and contextual factors. Universitas Psychologica, 11, 293-309. https:// doi.org/10.11144/Javeriana.upsy11-1.cpif

Torres-Coronas, T., \& Vidal-Blasco, M. A. (2015). Students and employers perception about the development of digital skills in Higher Education. Revista de Educación, 367, 63-90. http://doi.org/10.4438/1988592X-RE-2015-367-283 